\title{
LOT SIZING AND CUTTING STOCK PROBLEMS IN A PAPER PRODUCTION PROCESS
}

\author{
Livia Maria Pierini ${ }^{1 *}$ and Kelly Cristina Poldi ${ }^{2}$
}

Received March 13, 2020 / Accepted November 11, 2020

\begin{abstract}
In many manufacturing industries, the production process involves the production of objects and the cutting of such objects into smaller pieces in order to meet a specific demand. In the optimization of these processes, one can identify the lot sizing and the cutting stock problems. In the literature, these problems are mostly separately dealt. However, treating these two problems in an integrated approach can decrease overall costs. In this research, we deal with the coupled lot sizing and cutting stock problem. It is proposed a mathematical formulation for the production and cutting of paper based on a real case, which considers setup costs and limited machine capacity in the production process of the objects. For the solution of the proposed model, we used a column generation approach and a relax-and-fix heuristic. Computational tests were carried out in order to analyze the methodology used in the resolution of the model. The results showed to be competitive in a reasonable computational time.
\end{abstract}

Keywords: cutting stock problem, lot sizing problem, integrated, Relax-and-Fix heuristic, column generation, paper industry.

\section{INTRODUCTION}

Since the industrial revolution, competitiveness among industries has triggered the search for production processes optimization aiming at the increase of productivity, avoiding waste and reducing costs. Particularly in times of crisis, the demand for more efficient procedures becomes even greater since raising productivity and lowering costs increase profitability and, consequently, the ability to stay and grow in the market.

In many manufacturing industries, such as paper, furniture, and metallurgy, large objects are produced and then cut into smaller units to meet a given demand. In the optimization of such productive processes, the lot sizing and the cutting stock optimization problems arise.

\footnotetext{
*Corresponding author

${ }^{1}$ Departamento de Matemática Aplicada, IMECC, Universidade Estadual de Campinas, Rua Sérgio Buarque de Holanda, 651, 13083-859, Campinas, SP, Brasil - E-mail: liviam.pierini@ gmail.com - https://orcid.org/0000-0002-1469-7596

2 Departamento de Matemática Aplicada, IMECC, Universidade Estadual de Campinas, Rua Sérgio Buarque de Holanda, 651, 13083-859, Campinas, SP, Brasil - E-mail: kellypoldi@ime.unicamp.br - https://orcid.org/0000-0002-1649-6843
} 
The Lot Sizing Problem (LSP) aims to determine the number of items to be produced within a given time interval and how this production must occur in order to meet the demand and satisfy some optimization criteria. In the literature, many papers address the LSP in different environments, proposing mathematical models and solving them by exact and/or heuristic methods. Some reviews can be found in Jans and Degraeve (2008), Glock, Grosse and Ries (2014), Copil et al. (2017), Brahimi et al. (2017) and Doostmohammadi and Akartunali (2018).

The Cutting Stock Problem (CSP) determines how larger objects must be cut into smaller items in order to meet the demanded items and satisfy some optimization criteria. A CSP is usually modeled as an integer linear programming problem and its resolution comprises two main difficulties. The first one is related to the integrality constraints on the decision variables. A very simple way to overcome this difficulty is to relax the integrality constraints and then, employing some heuristics, an integer solution to the problem can be found. The second difficulty is due to a large number of variables, which can be bypassed by using a column generation approach. For more details related to the CSP, Wäscher, Haubner and Schumann (2007), Morabito, Arenales and Yanasse (2009), Song and Bennell (2014), Gomes et al. (2016) and Delorme, Iori and Martello (2016) are recommended.

Although the cutting stock problem is considered a fundamental subproblem of the production planning problem in many industries, these two problems have been handled in a decoupled way. However, in the last decades, according to Poltroniere et al. (2008), the integrated problem has become a trend and several models and solution methods proposals have been developed. It is possible to observe that better results can be obtained through integrated models (Gramani, França and Arenales (2009) and Vanzela et al. (2017)). In addition, some techniques present better results when applied to certain problems, which motivates new approaches with different models and combining different techniques. Thus, these techniques usually are developed for each specific case. Among the studies that address the integrated problem are Kantorovich (1960), Farley (1988), Poldi and Arenales (2010), Gramani and França (2006), Alem, Morabito and Ferreira (2013), Silva, Alvelos and Valério de Carvalho (2014), Melega, Araujo and Jans (2016), Wu, Akartunali and Jans (2017) and Ma et al. (2018). For more details, Melega, Araujo and Jans (2018) made a classification and literature review of integrated lot sizing and cutting stock problems.

Some papers specifically address the integrated problem in paper industries. Correia, Oliveira and Ferreira (2004) studied the integrated problem and proposed a heuristic solution method involving the simplex method with column generation and a rounding heuristic procedure. Poltroniere et al. (2008) proposed a model and two heuristic solution methods based on Lagrangian relaxation. Poltroniere, Araujo and Poldi (2016) made a computational study using heuristics and the CPLEX optimizer and involving two models: a new proposed model and the model proposed by Poltroniere et al. (2008). Leão, Furlan and Toledo (2017) proposed different mathematical reformulations for the integrated problem proposed by Poltroniere et al. (2008) and solved them using the column generation method and an adaptive large neighborhood search heuristic. Also based on the model proposed by Poltroniere et al. (2008), Campello et al. (2019) studied the inte- 
grated problem with a multiobjective approach by investigating correlations among the multiple objectives.

It is possible to observe in these studies that to solve integrated models, due to their complexity, different techniques for each case are combined and applied, such as several heuristics and column generation methods. Moreover, most of these studies consider the production of different types of paper in the same reel. Only Correia, Oliveira and Ferreira (2004) present the idea of separating the order according to the paper type. However, the authors just discussed general ideas of the constraints and do not present a mathematical model. Besides, the single objective of the study is to reduce the paper waste in the cutting process.

During the change of paper type in the production process occurs material waste, which could be avoided with the production of each paper type separately. This goal was observed in the paper industry and encouraged this research. Based on it, an integrated model for the lot sizing and cutting stock problems, which considers production costs, setup costs in the production of reels, inventory costs and limited machine capacity in the production process, is proposed to take into account the production of reels of each paper type separately. In the model resolution, a column generation approach and a relax-and-fix heuristic are applied. The proposed solution method was implemented and solved by CPLEX. Computational tests were carried out on randomly generated instances and, finally, an analysis of the obtained results is presented.

The paper is presented as follows. In Section 2, the proposed mathematical model is introduced. In Section 3 and 4, both adopted methods to solve the proposed model are presented and, then, the solution approaches are described in Section 5. The computational tests are discussed in Section 6 and, finally, some considerations and future proposals are presented in Section 7.

\section{PROBLEM FORMULATION}

In paper industries, large reels of different lengths and types of paper, called jumbos, are produced and cut in order to meet items' demand. In paper production, a jumbo may be composed of different types of paper, so during its production, one or more changes on the paper type occur and, consequently, there is paper waste in this process, as reported in Poltroniere et al. (2008). However, in order to decrease the material waste, in the studied paper industry, the jumbo production of each type of paper occurs separately, i.e., each roll of paper produced is composed of a single type. Moreover, in some industries, the paper produced is stored in warehouses until the withdrawal of the product by the customer, with a set deadline. In case of delay, the additional cost of stocking the items is paid by the customer. In this context, the integrated problem can be modeled in such a way as to consider paper waste and items inventory costs in the cutting process and production, setup and reels inventory costs in the production process.

In this context, the LSP determines the weight of the jumbos produced in each period of the planning horizon. The jumbo length and maximum weight depend on the machine on which it was produced. Also, demanded items can be obtained by the cutting of different jumbos. Thus, in this process, the number of produced jumbos of each weight and in each machine is determined. 
The CSP determines the number of jumbos that will be cut into smaller reels and the way it will be cut in each period of the planning horizon in order to meet demand with the minimum material waste. Figure 1 illustrates this process with four different cutting patterns and one period of the planning horizon in which seventeen jumbos must be produced and cut six times using the first cutting pattern, four times using the second cutting pattern, five times using the third cutting pattern and two times using the fourth cutting pattern.
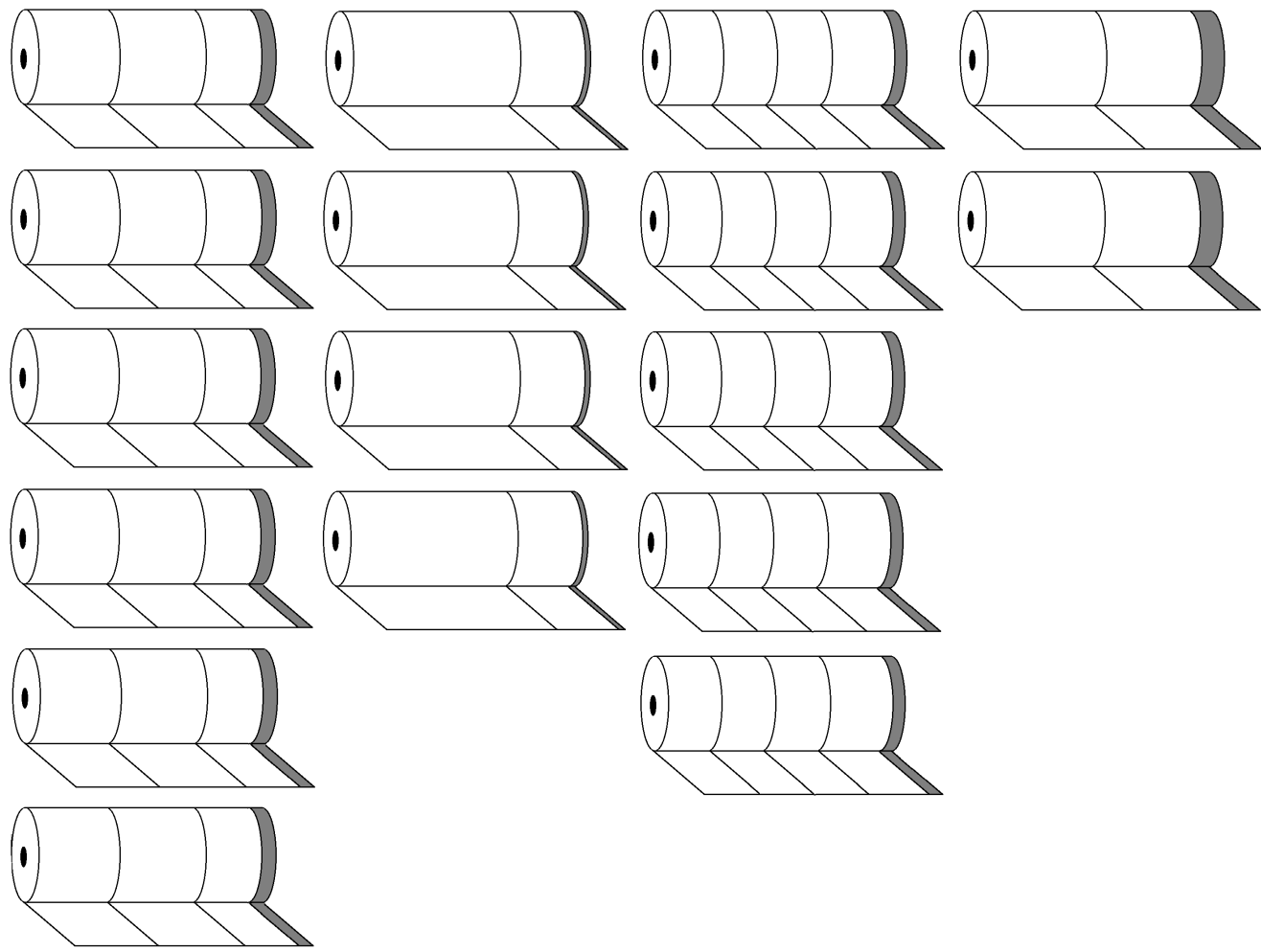

Figure 1 - Quantities of produced jumbos and the way to cut them into smaller reels.

The proposed model considers the jumbo type $m$ of length $L_{m} \mathrm{~cm}$ as a number of smaller reels of weight $b_{m} \mathrm{~kg}$. Therefore, supposing that $x_{m}$ is the number of smaller reels of length $L_{m} \mathrm{~cm}$ that must be produced, then the jumbo weight, $T_{m}$, is given by $T_{m}=L_{m} b_{m} x_{m}$. There is a limit of smaller reels that represents a jumbo since the jumbos have a maximum weight. Therefore, although the decision variables are integer in the formulation, the discretization of the jumbo into smaller reels tends to represent the jumbo continuously.

In order to simplify the notation, the smaller reels are denoted only by reels. For this formulation, it is important to know the weight of each object since the machine capacity is given as a function of weight. The proposed model for the production and cutting of paper is presented below. For this, consider the following data and parameters. 
Indices:

$t=1, \ldots, T:$ number of period in the planning horizon;

$m=1, \ldots, M$ : number of machines;

$j=1, \ldots, N_{m}$ : number of cutting pattern for reels of type $m ;$

$i=1, \ldots, N$ : number of ordered item.

Parameters:

$s_{m t}:$ setup cost for machine $m$ producing a reel in period $t$;

$c_{m t}$ : production cost for a reel being made in machine $m$ in period $t$;

$h_{t}$ : inventory cost for a reel at the end of period $t$;

$\sigma_{i t}$ : cost for holding final items $i$ at the end of period $t$;

$c p_{t}$ : cost for each centimeter of paper lost during the cutting process in period $t$;

$b_{m}$ : weight of reel produced in machine $m$;

$f_{m}$ : paper lost (ton) in setting up machine $m$;

$\eta_{i}$ : weights of final items $i$;

$p_{j m}$ : paper waste $(\mathrm{cm})$ in cutting pattern $j$ used to cut a reel of length $L_{m}$;

$O_{t}$ : demand (ton) of paper in period $t$;

$C_{m t}$ : capacity (ton) of machine $m$ in period $t$;

$a_{i j m}$ : number of items $i$ cut according to cutting pattern $j$ from the reel of length $L_{m}$;

$d_{i t}$ : demand of final item type $i$ in period $t$;

Variables:

$x_{m t}:$ number of reels produced in machine $m$ in period $t$;

$w_{m t}$ : number of reels produced in machine $m$ stored at the end of period $t$;

$z_{m t}$ : binary variable that means if there is production or not in machine $m$ in period $t$;

$y_{m t}^{j}:$ number of reels produced in machine $m$ in period $t$ which are cut using the cutting pattern $j$;

$e_{i t}$ : number of final items type $i$ held at the end of period $t$; 
Therefore, the proposed integrated model is given by:

$$
\begin{aligned}
\min & \sum_{t=1}^{T} \sum_{m=1}^{M}\left(c_{m t} x_{m t}+h_{t} b_{m} w_{m t}+s_{m t} z_{m t}\right)+ \\
& \sum_{t=1}^{T} \sum_{m=1}^{M} \sum_{j=1}^{N_{m}} c p_{t} p_{j m} y_{m t}^{j}+\sum_{t=1}^{T} \sum_{i=1}^{N} \sigma_{i t} \eta_{i} e_{i t} \\
\text { s.t. } & \sum_{m=1}^{M}\left(b_{m} x_{m t}+b_{m} w_{m, t-1}-b_{m} w_{m t}\right)=O_{t}, \quad t=1, \ldots, T \\
& b_{m} x_{m t} \leq\left(f_{m}-C_{m t}\right) z_{m t}, \quad m=1, \ldots, M ; t=1, \ldots, T \\
& \sum_{m=1}^{M} \sum_{j=1}^{N_{m}} a_{i j m} y_{m t}^{j}+e_{i, t-1}-e_{i, t}=d_{i t}, \quad i=1, \ldots, N ; t=1, \ldots, T \\
& \sum_{j=1}^{N_{m}} y_{m t}^{j}=x_{m t}+w_{m, t-1}-w_{m t}, \quad m=1, \ldots, M ; t=1, \ldots, T \\
& w_{m 0}=0, e_{0}=0, m=1, \ldots, M \\
& x_{m t} \in \mathbb{Z}^{+}, w_{m t} \in \mathbb{Z}^{+}, \quad m=1, \ldots, M ; t=1, \ldots, T \\
& y_{m t}^{j} \in \mathbb{Z}^{+}, e_{i t} \in \mathbb{Z}^{+}, \quad j=1, \ldots, N_{m} ; m=1, \ldots, M ; t=1, \ldots, T ; i=1, \ldots, N \\
& z_{m t} \in\{0,1\}, \quad m=1, \ldots, M ; t=1, \ldots, T .
\end{aligned}
$$

The objective function (1) is composed of three terms and it aims to minimize production and cutting process costs. The first term aims to minimize the production and inventory cost of reels and the setup cost. The second term seeks to minimize the total waste of material in the cutting process. The last term of the objective function seeks to minimize the inventory cost of final items. Note that the considered setup cost in the formulation is related to the setting up of the machine for the production of objects.

The constraints (2) are the inventory balancing constraints, i.e., they ensure that the total amount of paper produced in each period plus the stock of the previous period minus the stock of the current period must be equal to the demand of paper. The parameter is determined as a dependent variable given by $O_{t}=\sum_{m=1}^{M} \sum_{j=1}^{N_{m}} b_{m} y_{m t}^{j}$ in order to optimize the paper demand and minimize the waste paper instead of estimating it. Therefore, the solution heuristic proposed by Poltroniere et al. (2008) does not apply to our model since it depends on the paper demand estimated before the model resolution.

The constraints (3) ensure that, for each machine and period of the planning horizon, the total amount of produced paper does not exceed the capacity of the machine.The constraints also associate the binary variable $z_{m t}$ with the period and machine production, i.e., $z_{m t}=1$ when $x_{m t}>0$ and, in the case that $x_{m t}=0$, the optimality results in $z_{m t}=0$.

The constraints (4) are the item inventory balancing constraints, i.e., they describe that, in each period and for each item, the total quantity of each cut item plus the number of stored items from the previous period less the stock of items in the current period must be equal to the demand for 
items. The constraints (5) are the coupling constraints, that is, they associate the production and the cutting of reels. These constraints ensure that, for each machine and period, the total amount of cut reels is equal to the quantity of produced reels plus the stock of the previous period less the stock of the current period. These constraints consider the possibility of stocking the reels and cutting them in later periods.

In constraints (6), it is considered that the initial stock of reels and items are null. If the initial stock is different from zero, it suffices to reduce the demand for the first periods until it becomes null. The constraints (7) and (8) are non-negativity and integrality of the model variables. The constraints (9) ensure that the variables $z_{m t}$ are binary.

\section{THE COLUMN GENERATION APPROACH}

One of the difficulties encountered in solving a CSP is the number of variables, which increases as the amount of cutting patterns is greater. The number of possible columns in the CSP can be very large, making it almost impossible to analyze all the columns of the problem. Thus, the approach consists of generating promising columns to get into the base.

The technique was introduced by Gilmore and Gomory (1961), who proposed a modification of the simplex method and which consists of solving the relaxed problem with only a subset of known cutting patterns. For this, at each iteration, cutting patterns are generated in order to improve the current solution of the relaxed problem, until obtaining the optimal solution. In order to generate these cutting patterns, at each iteration, a subproblem must be solved. In this sense, the vector $a_{j m}$ associated to the cutting pattern $j$ for reel of length $L_{m}$ is the vector of subproblem variables and each component $a_{i j m}$ means the number of items $i$ cut according to cutting pattern $j$ for the reel of length $L_{m}$.

Let $A$ be the constraint coefficient matrix, $B$ the basic partition of the matrix $A$ and $\pi$ the simplex multiplier vector, that is, $\pi^{T}=c_{B}^{T} B^{-1}$, where $c_{B}$ is the cost vector $c_{j}$ associated with the basic variables. Let $\ell_{i}$ be the length of the item type $i, L_{m}$ the length of the object type $m, m=1, \ldots, M$, and $N$ the number of item types. Thus, in the case of the one-dimensional CSP with $M$ types of objects to be cut, for each object $m$, the subproblem is given by:

$$
\begin{array}{ll}
\text { minimize } & c p_{t} p_{j m}-\pi^{T} a_{j m} \\
\text { subject to: } & \sum_{i=1}^{N} \ell_{i} a_{i j m} \leq L_{m}, \\
& a_{i j m} \in \mathbb{Z}^{+}, \quad i=1, \ldots, N .
\end{array}
$$

The objective function (10) looks for the variable, that is, the cutting pattern, with the lowest relative cost, guaranteeing, when entering the base of the problem, a better solution. The constraints (11) and (12), which characterize a knapsack problem, ensure that the sum of the length of the items that compose the cutting pattern does not exceed the object size to be cut and that the quantities of cut items are non-negative. 
Note that a column of the constraint matrix of the model (1)-(9), and consequently of the subproblem, referring to the cutting pattern $j$ associated with the object $m$ is of the form $a_{j m}^{T}=\left(a_{1 j m}, a_{2 j m}, \ldots, a_{N j m}, 0, \ldots, 1,0, \ldots, 0\right)$, with 1 in the position $N+m$, due to the coupling constraints. Thus, when considering the value of $c p_{t} p_{j m}$, the objective function (10) of the subproblem can be expressed by:

$$
c p_{t} p_{j m}-\pi^{T} a_{j m}=c p_{t}\left(L_{m}-\sum_{i=1}^{N} \ell_{i} a_{i j m}\right)-\sum_{i=1}^{N} \pi_{i} a_{i j m}-\pi_{N+m} .
$$

This procedure then consists of generating a column $j$, that is, a cutting pattern $a_{j}$, looking for the variable with the lowest relative cost. Thus, for each object, we have a subproblem, equivalent to the knapsack problem, in the one-dimensional case.

The initial basic matrix of the simplex method of the CSP can be constructed with the homogeneous cutting patterns referring to the different objects available in stock. Homogeneous cutting patterns are patterns that produce only one type of item. In cases where there is a limitation on the number of stocked objects, the initial homogeneous solution may be infeasible and phase one of the simplex method must be performed, which consists of obtaining an initial feasible solution through introducing artificial non-negative variables to the model and solving it minimizing these variables value.

Note that the column generation method is used for the CSP with the relaxed integrality of the variables constraints. Thus, in order to find an integer solution to the problem, heuristic rounding methods can be applied. Such heuristics are found in Stadtler (1990), Wäscher and Gau (1996), Poldi and Arenales (2006), Poldi and Araujo (2016), among others.

\section{RELAX-AND-FIX HEURISTIC}

A very well known and widely used heuristic for mixed-integer linear programming problems is the relax-and-fix heuristic (Pochet and Wolsey (2006)). In the literature, it is possible to find several researches that use this heuristic in solving the lot sizing problem, such as Stadtler (2003), Mercé and Fontan (2003) and Toledo et al. (2015).

The relax-and-fix heuristic is based on dividing a problem, with integer variables, into several simpler subproblems. For this, the set of integer variables is divided into $P$ distinct subsets, $R_{k}$, $k=1, \ldots, P$. The number of iterations of the heuristic is determined by the number of subsets $P$. In each iteration $s$, the variables of the set $R_{s}$ are defined as integers, the variables of the sets $R_{k}, k=1, \ldots, s-1$, are fixed and the variables of the sets $R_{k}, k=s+1, \ldots, P$, are relaxed, and the subproblem is solved. If it is infeasible, the process is interrupted because it is not possible to find a solution to the problem with the variables belonging to the sets $R_{k}, k=1, \ldots, s-1$. Otherwise, the variables of the set $R_{S}$, or part of them, are fixed. Then, the process repeats, until all variables have an integer value. 


\section{METHODOLOGY}

The proposed integrated model (1)-(9), the column generation method and the relax-and-fix heuristic were both implemented in $\mathrm{C}++$ using the Visual Studio 2015 and the ILOG Concert Technology interface from CPLEX 12.10 on an Intel Core i7 computer with $3.6 \mathrm{GHz}$ and 16 Gbytes of memory.

At first, the column generation method was applied to the model with the relaxed integrality of the variables constraints (7)-(9). Thus, in this step, all the integer variables were considered real positive numbers and the binary variables considered real variables with values between 0 and 1. At this stage, cutting patterns were generated until an optimal solution to the relaxed problem was found.

In order to start the column generation method, a set of homogeneous cutting patterns was used for each type of object. Thus, for each item $i$, the quantity produced by the homogeneous cutting pattern $j$ referring to the cut of the object type $m$ was determined by $a_{i j m}=\left\lfloor L_{m} / \ell_{i}\right\rfloor$, that is, the largest integer value less than $L_{m} / \ell_{i}$.

Then, with the set of cutting patterns determined at the end of the column generation, the relaxand-fix heuristic was applied to the relaxed model, in order to obtain an integer solution. The set of variables was divided according to each period. Therefore, in the first iteration of relaxand-fix heuristic, the variables from period 1 were considered as integer and the remaining ones were determined real. The model was solved and, in the following iteration, the solution of the variables from period 1 was fixed, the variables from period 2 were considered the integer ones and the model was solved again. Then, the solution of the variables from period 2 was fixed and the variables from period 3 were considered the integer ones. This procedure was repeated successively until obtaining an integer solution for the problem.

At each iteration of relax-and-fix heuristic, the time to solve the problem was limited to 60 seconds. In order to avoid infeasibility, a strategy to modify some fixed variables was used. If the model was infeasible at iteration $s$, then the values fixed in the previous iteration $s-1$ were considered a lower bound to these variables and the problem was solved again. If the CPLEX optimization package found a feasible solution, then the integer variables from iteration $s$ and $s-1$ were fixed in their current solution and the heuristic proceeded as described in the previous paragraph. If the model was still infeasible, then the strategy was applied also for the variables from iteration $s-2$, i.e., the values fixed in the iteration $s-2$ became a lower bound to these variables and the model was solved over again. The strategy was applied $r$ times, until obtaining a feasible solution or $s-r \leq 1$.

In order to analyze the necessity and advantages of the applied relax-and-fix heuristic, the model (1)-(9) was also solved by CPLEX with the generated columns determined at the end of the column generation, with a time limit of 600 seconds and CPLEX tolerance gap fixed in $10^{-3}$. 


\section{COMPUTATIONAL TESTS}

Table 1 shows the set of data used. The first column (Parameter) introduces parameters' identifications and the second column (Value) shows the considered values.

Table 1 - Input Data.

\begin{tabular}{|c|c|}
\hline Parameter & Value \\
\hline Weight of produced reel & $b_{m}=L_{m} \rho$ \\
\hline Production cost of reel & $c_{m t} \in\left[\begin{array}{ll}0.015 & 0.025\end{array}\right] b_{m}$ \\
\hline Setup cost & $s_{m t} \in\left[\begin{array}{ll}0.03 & 0.05\end{array}\right] c_{m t}$ \\
\hline Wasted paper in setting up the machine & $f_{m} \in\left[\begin{array}{ll}0.01 & 0.05\end{array}\right] b_{m}$ \\
\hline Inventory cost for reel & $h_{t} \in\left[\begin{array}{ll}0.0000075 & 0.0000125\end{array}\right]$ \\
\hline Inventory cost for final items & $\sigma_{i t}=0.5 \cdot h_{t}$ \\
\hline Trim loss cost in the cutting process & $c p_{t}=\frac{\sum_{m=1}^{M} c_{m t}}{M} \cdot 10$ \\
\hline Items length & $l_{i} \in\left[\begin{array}{ll}0.1 & 0.3\end{array}\right] \cdot \frac{\sum_{m=1}^{M} L_{m}}{M}$ \\
\hline Demand of final items & $d_{i t} \in\left[\begin{array}{ll}0 & 300\end{array}\right]$. If $d_{i t} \leq 50$, then $d_{i t}=0$ \\
\hline Capacity of the production machine & $C_{m t}=\frac{b_{m}}{\sum_{m=1}^{M} b_{m}} \cdot \alpha \frac{\sum_{t=1}^{T} \sum_{m=1}^{M}\left(\frac{D_{t}}{M}+f_{m}\right)}{T}$ \\
\hline
\end{tabular}

The data set used in the computational experiment was based on Poltroniere et al. (2008). The number of demanded items was considered $N=5,10$ and 20 and the number of periods was determined by $T=8,10$ and 12 . The number of machines and the specific weight (density) of the reels in all instances remained constant, $M=2$ and $\rho=2 \mathrm{~kg} / \mathrm{cm}$ respectively, with the machine 1 producing jumbos of length $L_{1}=540 \mathrm{~cm}$ and the machine 2, jumbos of length $L_{2}=460 \mathrm{~cm}$. The capacity of the production machine was considered in two distinct scenarios: Normal Capacity $(N C)$, based on Poltroniere et al. (2008) with $\alpha=1.24$, and Tight Capacity (TC), considering $\alpha=1.17$. Any value for alpha lower than 1.17 led to infeasible instances. Therefore, 18 distinct classes were generated with 10 instances in each class. The proposed test instances are available at http://www.ime.unicamp.br/ kelly/PO2021/. 


\subsection{Computational Results}

The performed computational tests and the analysis of the results are presented. Table 2 shows the average number of iterations, generated columns and spent time by applying the column generation method in the model (1)-(9) with the relaxed integrality constraints (7)-(9). In the first column (Class), the classes are identified. The second column contains the information of the number of periods $(T)$ and items $(N)$ and type of machine capacity $(C)$ of each class. Then, the average number of iteration and generated columns for each class is presented. Finally, the last column shows the average time spent by the column generation method.

Table 2 - Average number of iteration and generated columns when applying the column generation method for each class of instances.

\begin{tabular}{ccrrr}
\hline Class & $T / N / C$ & Iteration & Generated Col. & GC Time \\
\hline 1 & $08 / 05 / N C$ & 5.7 & 12.0 & 2.6 \\
2 & $08 / 10 / N C$ & 11.2 & 43.6 & 2.3 \\
3 & $08 / 20 / N C$ & 19.2 & 95.9 & 4.4 \\
4 & $10 / 05 / N C$ & 5.6 & 12.6 & 1.4 \\
5 & $10 / 10 / N C$ & 12.3 & 43.2 & 3.2 \\
6 & $10 / 20 / N C$ & 18.8 & 86.9 & 5.8 \\
7 & $12 / 05 / N C$ & 5.5 & 14.7 & 1.3 \\
8 & $12 / 10 / N C$ & 11.8 & 48.0 & 3.7 \\
9 & $12 / 20 / N C$ & 18.0 & 101.2 & 6.5 \\
10 & $08 / 05 / T C$ & 4.8 & 9.8 & 0.9 \\
11 & $08 / 10 / T C$ & 12.4 & 42.4 & 2.7 \\
12 & $08 / 20 / T C$ & 20.5 & 78.3 & 5.3 \\
13 & $10 / 05 / T C$ & 5.2 & 13.4 & 1.0 \\
14 & $10 / 10 / T C$ & 11.8 & 39.8 & 2.9 \\
15 & $10 / 20 / T C$ & 17.3 & 82.1 & 5.6 \\
16 & $12 / 05 / T C$ & 5.1 & 12.4 & 1.2 \\
17 & $12 / 10 / T C$ & 12.6 & 40.4 & 3.6 \\
18 & $12 / 20 / T C$ & 18.0 & 88.2 & 7.0 \\
\hline & Average & 12.0 & 48.1 & 3.4 \\
\hline
\end{tabular}

The number of generated columns, that is, generated cutting patterns, on average, varied from 9.8, for instances with 5 items and 8 periods and tight capacity, to 101.2, for instances with 20 items and 12 periods and normal capacity. The column generation method presented 12 iterations and spent 3.4 seconds on average. At each iteration of the column generation method, all the columns found with negative relative cost were considered to enter at the base of the problem in order to reduce the number of iterations and also to obtain a greater number of generated columns for the integer solution finding step. It explains the greater number of columns when compared with the number of iteration. It is also possible to observe that the solution methods had a similar performance for both normal and tight capacity scenarios. The average used capacity was $80.8 \%$ 
for instances with normal capacity and $85.7 \%$ for instances with tight capacity for both solution methods.

In Table 3, the average gaps of the relax-and-fix heuristic and CPLEX applications are shown for each class. In the first and second columns, the classes, the number of periods (T) and items (N) and the type of machine capacity (C) of each set of instances are identified. The RF Gap column shows the average gap obtained at the end of the relax-and-fix heuristic application. The RF Gap was calculated by the difference between the objective function obtained at the end of relaxand-fix heuristic application $(\mathrm{FH})$ and the value of the objective function obtained at the end of the column generation method (FM), divided by $F M$, times 100 , i.e., RF Gap $=100 \frac{F H-F M}{F M}$. The CPLEX Gap column presents the average gap of CPLEX solution and was calculated by the difference between the objective function obtained by CPLEX (FC) and the value of the objective function obtained at the end of the column generation method (FM), divided by $F M$, times 100, i.e., $\mathrm{RF}$ Gap $=100 \frac{F C-F M}{F M}$.

Table 3 - Average gap of relax-and-fix heuristic and CPLEX solutions for each class of instances.

\begin{tabular}{ccrr}
\hline Class & $T / N / C$ & RF Gap (\%) & CPLEX Gap (\%) \\
\hline 1 & $08 / 05 / N C$ & 0.09 & 0.10 \\
2 & $08 / 10 / N C$ & 0.07 & 0.11 \\
3 & $08 / 20 / N C$ & 0.05 & 0.10 \\
4 & $10 / 05 / N C$ & 0.09 & 0.09 \\
5 & $10 / 10 / N C$ & 0.05 & 0.09 \\
6 & $10 / 20 / N C$ & 0.03 & 0.09 \\
7 & $12 / 05 / N C$ & 0.13 & 0.14 \\
8 & $12 / 10 / N C$ & 0.05 & 0.08 \\
9 & $12 / 20 / N C$ & 0.04 & 0.11 \\
10 & $08 / 05 / T C$ & 0.16 & 0.09 \\
11 & $08 / 10 / T C$ & 0.09 & 0.12 \\
12 & $08 / 20 / T C$ & 0.03 & 0.10 \\
13 & $10 / 05 / T C$ & 0.07 & 0.08 \\
14 & $10 / 10 / T C$ & 0.09 & 0.13 \\
15 & $10 / 20 / T C$ & 0.04 & 0.10 \\
16 & $12 / 05 / T C$ & 0.09 & 0.10 \\
17 & $12 / 10 / T C$ & 0.05 & 0.10 \\
18 & $12 / 20 / T C$ & 0.04 & 0.10 \\
\hline & Average & 0.07 & 0.10 \\
\hline
\end{tabular}

Note that both methodologies presented small gaps and the RF Gap was smaller than the CPLEX gap. It may be noticed that the average values of the RF gaps decrease as the number of items increases for both normal and tight capacity, which does not happen with CPLEX solutions. Therefore, the difference between the gaps of both methodologies is greater for instances with 20 items, as one can see in Class 3, 6, 9, 12, 15 and 18, where the relax-and-fix heuristic application 
performed better. Furthermore, the gaps found for the normal and the tight capacities instances were very similar, ensuring the quality of the solution for both scenarios.

In Table 4, it is shown the computational times, reported in seconds, spent by the relax-andfix (RF Time) and CPLEX (CPLEX Time) applications. The total time, i.e., the sum of all the computational time is also presented for both methodologies (RF Total and CPLEX Total). Class column presents the class identity and $T / N / C$ column shows the number of periods and items and type of machine capacity of each class.

Table 4 - Average computational time, in seconds, for each class of instances, spent by both methodologies.

\begin{tabular}{ccrrrr}
\hline Class & $T / N / C$ & RF Time & CPLEX Time & RF Total & CPLEX Total \\
\hline 1 & $08 / 05 / N C$ & 0.3 & 0.1 & 2.9 & 2.6 \\
2 & $08 / 10 / N C$ & 0.3 & 0.4 & 2.6 & 5.1 \\
3 & $08 / 20 / N C$ & 0.8 & 28.1 & 5.2 & 35.4 \\
4 & $10 / 05 / N C$ & 0.7 & 0.1 & 2.2 & 1.3 \\
5 & $10 / 10 / N C$ & 25.0 & 0.4 & 28.3 & 3.8 \\
6 & $10 / 20 / N C$ & 1.2 & 111.1 & 7.0 & 118.5 \\
7 & $12 / 05 / N C$ & 0.4 & 0.1 & 1.7 & 1.6 \\
8 & $12 / 10 / N C$ & 0.5 & 0.5 & 4.2 & 4.3 \\
9 & $12 / 20 / N C$ & 1.4 & 105.9 & 7.9 & 114.8 \\
10 & $08 / 05 / T C$ & 0.5 & 0.0 & 1.4 & 0.9 \\
11 & $08 / 10 / T C$ & 0.3 & 0.3 & 3.0 & 3.7 \\
12 & $08 / 20 / T C$ & 0.9 & 65.0 & 6.2 & 72.4 \\
13 & $10 / 05 / T C$ & 0.3 & 0.1 & 1.3 & 1.3 \\
14 & $10 / 10 / T C$ & 0.4 & 0.5 & 3.3 & 4.1 \\
15 & $10 / 20 / T C$ & 1.2 & 25.6 & 6.8 & 33.4 \\
16 & $12 / 05 / T C$ & 0.4 & 0.1 & 1.5 & 1.7 \\
17 & $12 / 10 / T C$ & 0.5 & 0.5 & 4.1 & 5.0 \\
18 & $12 / 20 / T C$ & 1.4 & 23.4 & 8.4 & 33.5 \\
\hline & Average & 2.0 & 20.1 & 5.4 & 24.7 \\
\hline
\end{tabular}

The relax-and-fix heuristic application found feasible solutions for all the studied instances, while the CPLEX was not able to find a feasible solution in two instances of Class 9 and one instance of Class 18. Moreover, it is possible to observe that the time spent by CPLEX increases more than the relax-and-fix heuristic when the number of items increases, evidencing the advantages of the relax-and-fix heuristic application for instances with a large number of items.

Note that the average time spent in the relax-and-fix heuristic application was 2 seconds while CPLEX spent 20.1 seconds to solve the problem. Although CPLEX was faster for instances with the smallest number of items, the relax-and-fix heuristic was faster on average, mainly for instances with the biggest number of items. 
The total time spent to solve the problem by the relax-and-fix heuristic was on average 5.4 seconds while CPLEX took 24.7 seconds. At instance 9 of the Class 5 the relax-and-fix heuristic spent 246 seconds to finish, which increased the average of Class 5. However, all the other instances of Class 5 was solved in less than 0.5 seconds. Only 5 from the 180 studied instances spent more than 2 seconds to find an integer solution for the problem by the relax-and-fix heuristic.

Although, the relax-and-fix heuristic presented a similar spent time in the normal and tight capacity scenarios at most the classes, both methodologies spent less computational time, on average, for solving the instances with tight capacity. It is possible to observe that, for instances with 20 different items, CPLEX spent more time solving the normal capacity instances than the tight ones, indicating better performance in scenarios with tight capacity when there is a huge number of items.

\section{CONCLUSIONS AND FUTURE RESEARCH}

In this research, we propose an integrated model for lot sizing and cutting stock problems in the industrial paper production process, which considers limited machine capacity and setup costs in the production process of reels, inventory costs and production costs. In order to solve the mathematical model, we apply a column generation method to the relaxed model. Next, we use a relax-and-fix heuristic to find an integer solution for the problem. In order to analyze the relax-and-fix heuristic, the model was also solved by CPLEX with the generated columns. Computational tests were performed for 18 classes with 10 instances each. The number of periods was ranged from 8 to 12 , the number of items ranged from 5 to 20 and the machine capacity was considered in normal and tight scenarios.

The results showed that by using the applied column generation method and relax-and-fix heuristic, it is possible to solve the model obtaining good gaps in a small computational time since the average time spent to solve the instances was 5.4 seconds and the gap was, on average, $0.07 \%$. Both applied solution methods presented gaps smaller than $0.14 \%$, indicating the capacity of finding good solutions for both tight and normal capacity scenarios. The CPLEX application was faster for smaller instances. However, the relax-and-fix heuristic performed better, spending less time on average, mainly for bigger instances.

We consider that each produced reel has a unique type of paper. Therefore, in an industrial process involving the production and cutting of reels of different paper types, the integrated model should be solved specifically for each type of paper separately. As future research, it is intended to extend the model to consider multiple plants, i.e., the production of paper in factories of different locations. 


\section{Acknowledgements}

This research was funded by the Coordination for the Improvement of Higher Education Personnel - CAPES and the Sao Paulo Research Foundation - FAPESP (grants 2016/01860-1, 2017/18192-4).

\section{References}

[1] Alem D, Morabito R \& Ferreira JS. 2013. Risk-averse two-stage stochastic programs in furniture plants. OR Spectrum, 35(4): 773-806.

[2] Brahimi N, Absi N, Dauzère-PÉrès S \& Nordli A. 2017. Single-item dynamic lotsizing problems: An updated survey. European Journal of Operational Research, 263(3): 838-863.

[3] Campello BSC, Oliveira WA, Ayres AOC \& Ghidini CTlS. 2019. A multiobjective integrated model for lot sizing and cutting stock problems. Journal of the Operational Research Society, DOI: 10.1080/01605682.2019.1619892.

[4] Copil K, Wörbelauer M, Meyr H \& Tempelmeier H. 2017. Simultaneous lotsizing and scheduling problems: a classification and review of models. OR Spectrum, 39(1): $1-64$.

[5] Correia MH, Oliveira JF \& Ferreira JS. 2004. Reel and sheet cutting at a paper mill. Computers \& Operations Research, 31: 1223-1243.

[6] CPLEX 12.10. 2020. Users' manual and reference manual. ILOG S. A.

[7] Delorme M, IORI M \& Martello S. 2016. Bin packing and cutting stock problems: Mathematical models and exact algorithms. European Journal of Operational Research, 255(1): 1-20.

[8] Doostmohammadi M \& Akartunali K. 2018. Valid inequalities for two-period relaxations of big-bucket lot-sizing problems: Zero setup case. European Journal of Operational Research, 267(1): 86-95.

[9] FARLEY AA. 1988. Mathematical programming models for cutting-stock problems in the clothing industry. Journal of the Operational Research Society, 39(1): 41-53.

[10] Gilmore PC \& Gomory RE. 1961. A linear programming approach to the cuttingstock problem. Operations Research, 9(6): 849-859.

[11] Glock C, Grosse EH. And Ries JM. 2014. The lot sizing problem: A tertiary study. International Journal of Production Economics, 155: 39-51.

[12] Gomes AM, Gonçalves JF, Alvarez Valdés R \& Valério de Carvalho J. 2016. Preface to the Special Issue on Cutting and Packing. International Transactions in Operational Research, 23: 3-4. 
[13] GRAmani MCN \& FrançA PM. 2006. The combined cutting stock and lot-sizing problem in industrial processes. European Journal of Operational Research, 174: 509-521.

[14] Gramani MCN, França PM \& Arenales MN. 2011. A Lagrangian relaxation approach to a coupled lot-sizing and cutting stock problem. International Journal of Production Economics, 119: 219-227.

[15] Jans R \& Degraeve Z. 2008. Modeling industrial lot sizing problems: a review. International Journal of Production Research, 46(6): 1619-1643.

[16] Kantorovich LV. 1960. Mathematical methods of organizing and planning production. Management Science, 6(4): 366-422.

[17] Leão AAS, Furlan MM \& Toledo FM. B. 2017. Decomposition methods for the lot-sizing and cutting-stock problems in paper industries. Applied Mathematical Modelling, 48: 250-268.

[18] Ma N, LiU Y, Zhou Z \& ChU C. 2018. Combined cutting stock and lot-sizing problem with pattern setup. Computers \& Operations Research, 95: 44-55.

[19] Melega GM, Araujo SA \& Jans R. 2018. Classification and Literature Review of Integrated Lot-Sizing and Cutting Stock Problems. European Journal of Operational Research, 16: 1-19.

[20] Melega GM, Araujo SA \& Jans R. 2016. Comparison of MIP models for the integrated lot-sizing and one-dimensional cutting stock problem. Pesquisa Operacional, 36(1): 167-196.

[21] Mercé C \& FontAn G. 2003. MIP-based heuristics for capacitated lot sizing problems. International Journal of Production Economics, 85: 97-111.

[22] Morabito R, Arenales MN \& Yanasse HH. 2009. Special issue on cutting, packing and related problems. International Transactions in Operations Research, 16(6): 659.

[23] Pochet Y \& Wolsey LA. 2006. Production planning by mixed integer programming. New York: Springer.

[24] Poldi KC \& ARAujo SA. 2016. Mathematical models and a heuristic method for the multiperiod one-dimensional cutting stock problem. Annals of Operations Research, 238: 497-520.

[25] Poldi KC \& ARenales MN. 2006. Heurísticas para o problema de corte de estoque unidimensional inteiro. Pesquisa Operacional, 26(3): 473-492. 
[26] Poldi KC \& Arenales MN. 2010. O problema de corte de estoque unidimensional multiperíodo. Pesquisa Operacional (Impresso), 30: 153-174.

[27] Poltroniere SC, Poldi KC, Toledo FMB \& Arenales MN. 2008. A coupling cutting stock-lot sizing problem in the paper industry. Annals of Operations Research, 157: $91-104$.

[28] Poltroniere SC, Araujo SA \& Poldi KC. 2016. Optimization of an integrated lot sizing and cutting stock problem in the paper industry. Tendências em Matemática Aplicada e Computacional, 17(3): 305-320.

[29] Silva E, Alvelos F \& Valério de Carvalho JM. 2014. Integrating twodimensional cutting stock and lot-sizing problems. Journal of the Operational Research Society, 65: 108-123.

[30] Song X, \& Bennell JA. 2014. Column generation and sequential heuristic procedure for solving an irregular shape cutting stock problem. Journal of the Operational Research Society, 65(7): 1037-1052.

[31] Stadtler H. 1990. A one-dimensional cutting stock in the aluminium industry and its solution. European Journal of Operational Research, 44: 209-223.

[32] Stadtler H. 2003. Multilevel Lot Sizing with Setup Times and Multiple Constrained Resources: Internally Rolling Schedules with Lot-Sizing Windows. Operational Research, 51(3): 343-507.

[33] Toledo CFM, Arantes MS, Hossomi MYB, França PM \& Akartunal K. 2015. A relax-and-fix with fix-and-optimize heuristic applied to multi-level lot-sizing problems. Journal of Heuristic, 21(5): 687-717.

[34] Vanzela M, Melega GM, Rangel S, \& Araujo SA. 2017. The integrated lot sizing and cutting stock problem with saw cycle constraints applied to furniture production. Computers \& Operations Research, 79: 148-160.

[35] WÄSCHER G \& GAU T. 1996. Heuristics for the integer one-dimensional cutting stock problem: a computacional study. OR Spektrum, 18: 131-144.

[36] Wäscher G, Haubner H \& Schumann H. 2007. An improved typology of cutting and packing problems. European Journal of Operational Research, 183: 1109-1130.

[37] Wu T, Akartunali K, Jans R \& Liang Z. 2017. Progressive Selection Method for the Coupled Lot-Sizing and Cutting-Stock Problem. INFORMS Journal on Computing, 29(3): $377-580$.

\section{How to cite}

PIERINI LM \& POLDI KC. 2021. Lot sizing and cutting stock problems in a paper production process. Pesquisa Operacional, 41 (spe): e235094. doi: 10.1590/0101-7438.2021.041s1.00235094. 